\title{
Development of Driving Method for a Novel Motor without Rare Earth Materials
}

\author{
Hitoshi Miyata, Yuuki Sumida, Eikou Gonda \\ Department of Electrical and Computer Engineering \\ National Institute of Technology, Yonago College \\ 4448, Hikona-cho, Yonago-shi, Tottori-ken, 683-8502 Japan \\ Hitoshi Miyata: miyata@yonago-k.ac.jp
}

\begin{abstract}
This paper describes a novel motor which is an improved SRM (Switched Reluctance Motor) and its driving circuit. Our objective is applying the motor to the Electric Vehicles. In order to realize it, control method of the motor and driving circuits must be developed. For the control method, chopper, phase controller and inverter are proposed so far. On the other hand, SRM has strong nonlinearity and some disadvantages. Due to the nonlinearity, computer simulation of the motor and its driving circuits are quite difficult. Therefor we had to implement hardware and software to carry out experiment to evaluate our method. In this paper, at first, structural feature of the novel motor is explained. Then Investigation of phase control method for the motor also described. Finally, results of experiments of the motor drive by using driving circuits we developed will be presented.
\end{abstract}

Keywords: improved SRM, rare earth, EV.

\section{Introduction}

Hybrid and Electric Vehicle are receiving a lot of attention instead of Internal Combustion Engine Vehicle recently. In Japan, many automobile manufacturers have begun to make and sell Electric Vehicle (EV). Almost all motors applied to the EV, in Japan, are Interior Permanent Synchronous Motor (IPMSM) ${ }^{(1)}$. Neodymium which is one of the so called rare earth is employed as a magnet material of the IPMSM to realize high efficiency and high output. As rare earth is unevenly distributed in small amount on our planet, reliable supply is concerned in the near future. In addition, it is very expensive. Based on such a backdrop, we believe that applying the novel motor to $\mathrm{EV}$ enables us to produce EV at lower cost.

The driving method of the novel motor is similar to that of Switched Reluctance Motor (SRM). Disadvantages of the SRM are torque ripple, laud noise during the operation and so on. The new motor can be reduce those disadvantages by employing multilayer architecture. On the other hand, as driving method is under the study and the motor has strong nonlinear characteristics ${ }^{(2,3)}$, we have to assemble the driving circuit and study repeating its experiments.

There are three control method for the motor. That is to say, chopper, phase control and using inverter. In this study, we focused on the phase control method.

\section{Motor without Rare Earth Materials}

\subsection{Outline and Structure of the Motor}

The novel motor is a developed type of SRM. The motor can be reduce the noise, vibration and torque ripple. Fig. 1 indicates a cross section of the motor. Rotor and stator are

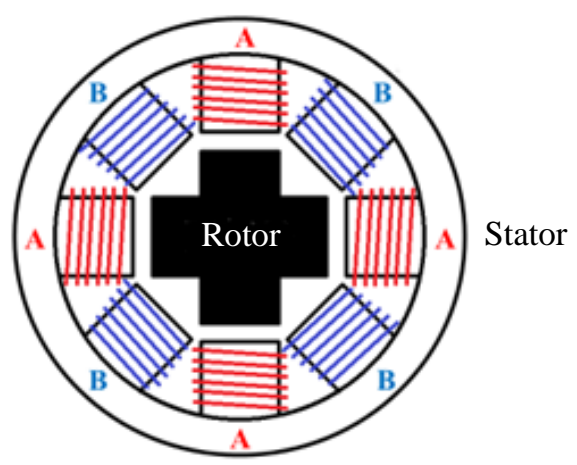

Fig. 1. A cross section of the novel motor. 


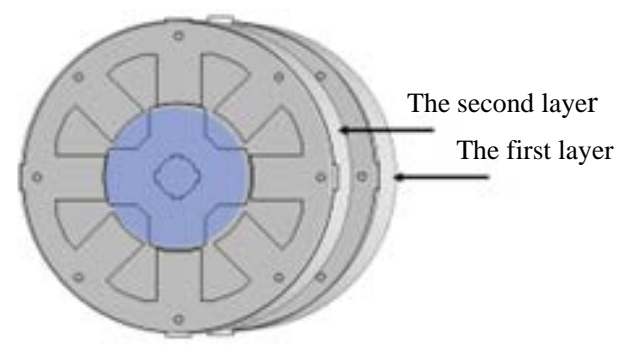

Fig. 2. A cross section of the novel motor.

made of electrical steel and both are salient pole structure. Only the stator has coil winding which is concentrated winding. The motor comprises 8 poles stator and 4 poles rotor. Fig. 1 illustrates the motor has 2 phase windings which include phase A winding and phase B winding. Fig. 2 shows the motor has a multilayered structure. In this case, stator and rotor are consist of multilayer components along the rotation axis direction.

\subsection{Reduction of Noise and Vibration}

In the case of conventional SRM, noise and vibration are occurred during operation. The cause of that phenomenon is compressive deformation. Fig. 3(a) illustrates compressive forces of SRM. The stator of the new motor is also deformed by attraction toward the rotor when the stator is exited. But as Fig. 3(b) shows, in the case of new motor, the forces of compressive deformation are mutually canceled. As a result, deformation of the stator which is the cause of the noise and vibration is considerably reduced.

\subsection{Multilayer Architecture}

The greatest feature of the new motor is a multilayer architecture as Fig. 4 shows. The conventional motor consists of only one layer stator and rotor. In the case of the new motor, two layers or more are laminated along the rotation axis direction. The second layer and after that are assembled rotating according to rotor angle of inclination. The rotor angle of inclination is different depending on the number of layers. This architecture enables to determine the rotation direction of the motor and to eliminate torque ripple.

Determination of the rotating direction is carried out as follows; Fig. 5 indicates only one layer of the stopping new motor whose phase A winding and salient pole of the rotor are in the confronting position. In this case, as current flows the winding of the painted pole, in Fig. 5, the rotor is applied clockwise torque and the stator is applied counterclockwise torque. As those torque cancels each other, the rotor never rotate. Fig. 6 illustrates multiple layer stator and position of

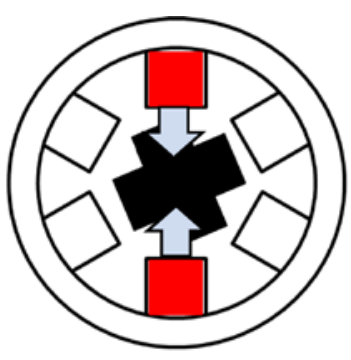

(a) SRM

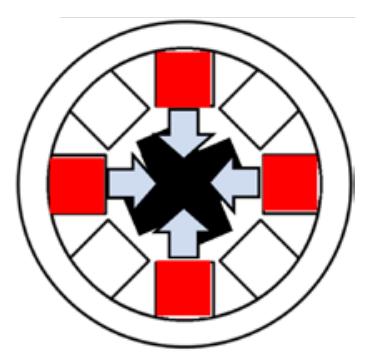

(b) The novel motor
Fig. 3. Force of compressive deformation of the stator.

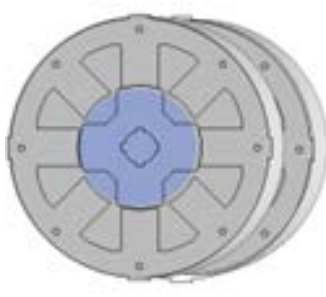

(a) Front view rotor angle of inclination

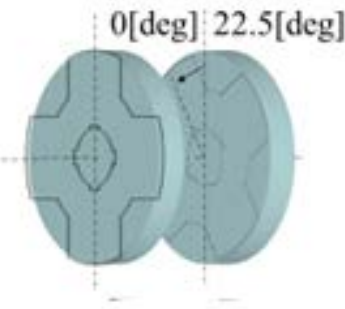

(b) Rotor angle of inclination
Fig. 4. A cross section of the novel motor.

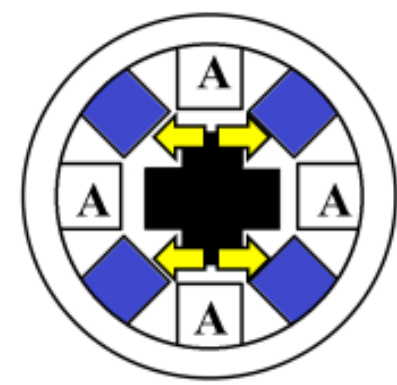

Fig. 5. In the case of the motor can't rotate.

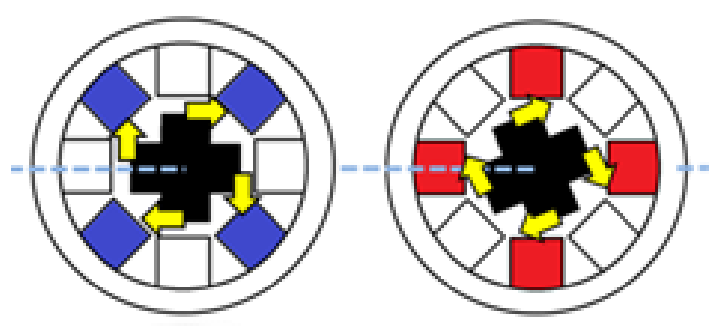

(a) The first layer

(b) The second layer

Fig. 6. Determination of the rotating direction. 


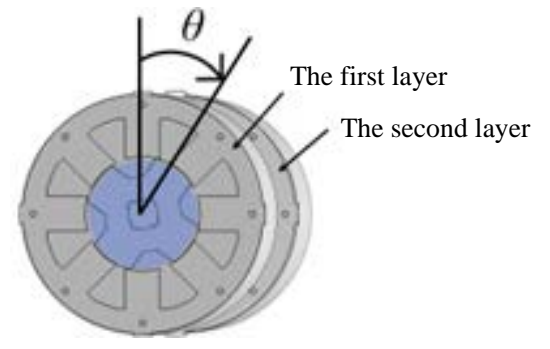

(a) Definition of $\theta$

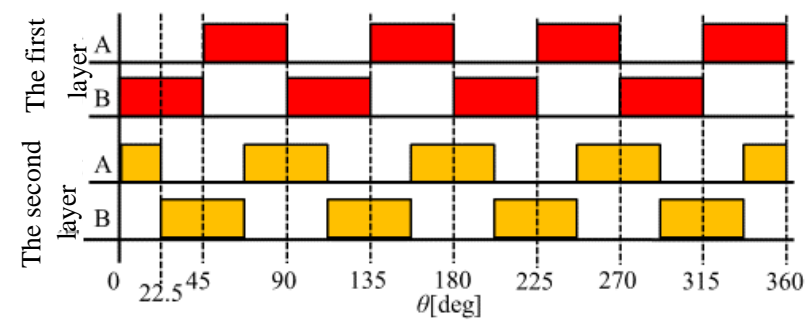

(b) Timing of the current

Fig. 7. Determination of the rotating direction.

the rotor and added second layer. At this time, position of the rotor of the second layer is in the middle of phase A winding and phase $B$ winding. As a result, in order to rotate the motor in the clockwise direction, the phase A winding painted in Fig. 6 is excited.

Fig. 7 illustrates in the case of the motor rotate in the clockwise. Fig. 7(a) indicates the definition of $\theta$. Fig. 7(b) designates the timing of current flows.

\section{Prototyping of Driving Circuits}

\subsection{Driving Circuits for the Novel Motor}

Driving circuit for the motor consists of motor driver and Digital Signal Processor (DSP). Control signal of the current is generated in the DSP by using information from current sensors. Signal from rotary encoder is also utilized in order to calculate the control signal.

\subsection{Test Equipment of the Novel Motor}

Test Equipment used in this study is indicated in Fig. 7. DC motor which is used for the load and the novel motor are jointed via coupling. Rotary encoder is fixed on the shaft of the DC motor. The load test is carry out by connecting resistance to the DC motor. The DC motor is operated as a generator to vary the load. The no load test is made by decoupling DC motor and the novel motor.

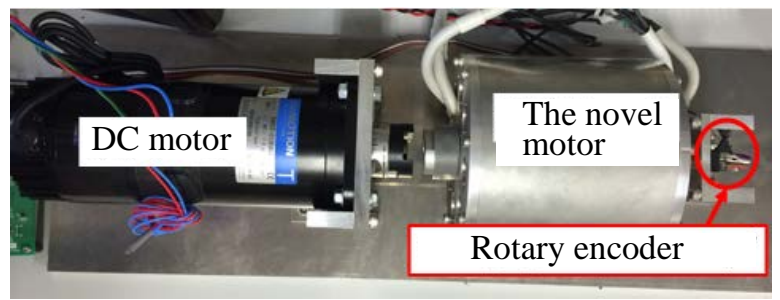

Fig. 8. Determination of the rotating direction.

\section{Results of Experiments}

\subsection{Test of Driving Circuit}

Experiments of driving circuit are no load test and speed control test. At first, in order to confirm the operation, the experiment was carried out with no load. After that, we made speed control test by using PI controller with varying load.

\subsection{No Load Test}

In this experiment, we measured power consumption of the driving circuits and the motor. Fig. 9 indicates experimental results. In Fig. 9, power consumption is rapidly increased from $3000[\mathrm{rpm}]$. It can be caused by time lag until sufficient magnetic flux was generated after exciting motor winding. That is to say, as the sufficient flux could not be generated at the time large amount of torque was required in the high speed operation, the motor was accelerate by using high power consumption. In order to improve that, motor winding is exited more quickly in conjunction with the motor speed is increase.

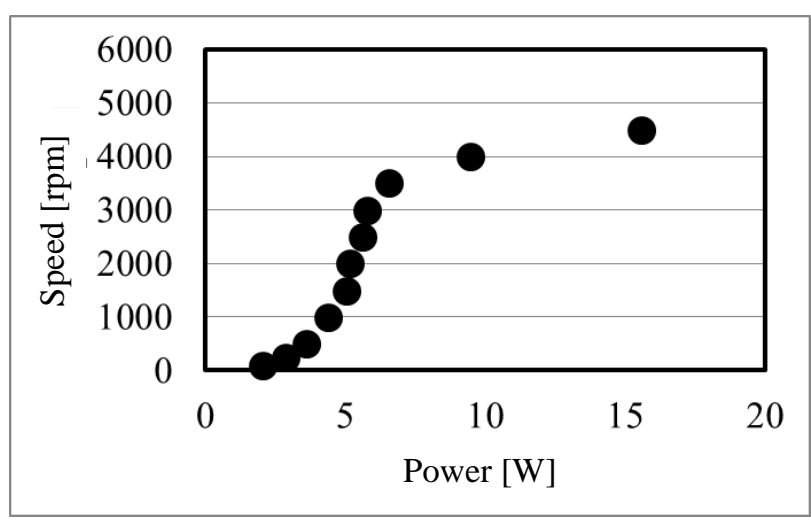

Fig. 9. Speed-Power characteristics. 


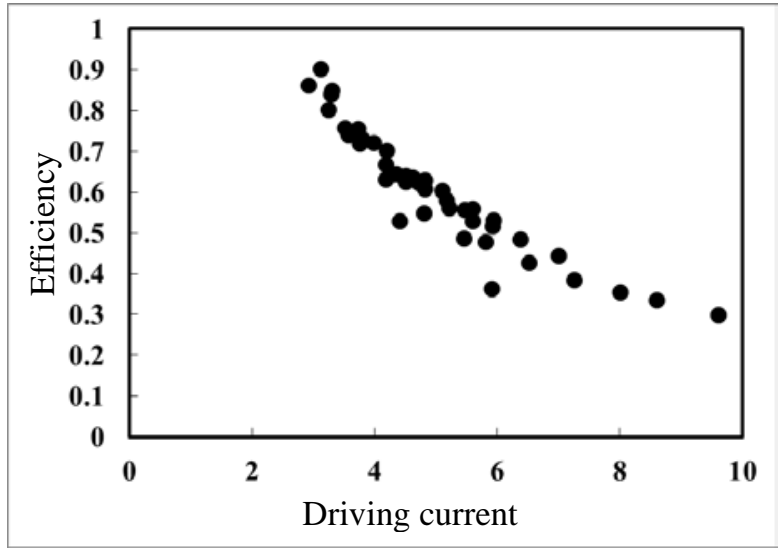

Fig. 10. Current-Efficiency characteristics.

\subsection{Load Test}

The load test was carried out by varying power consumption of load resistance. Result of the experiment is shown in Fig. 10. The efficiency in high load condition is reduced to under 50[\%]. Cause of this phenomenon has to be analyzed.

\section{Conclusions}

In this research, we developed the drive circuit for novel motor without rare earth materials. We have prototyped equipment and made some experiments. The motor have operated without fatal error. But the results of the experiments shows that there are room for improvement in some point of view. The more efficient operation method for the motor should be developed.

\section{References}

(1) Yoji Takeda, et al. : "Design and Control of Interior Permanent Synchronous Motor”, Ohmsha, pp. 2-3, 2001

(2) Koki Mastuse : "Control Engineering of Motors", Ohmsha, pp. 168, 2007

(3) Hisashi Takahashi and Takashi Yoshida : “The Latest Control Technic of Electric Vehicles”, NTS Inc., pp. 8182, 2011 